\title{
Preparation of Nano Ink Using Cobalt Ferrite and Barium Titanate for Printed Electronic Devices
}

\author{
Emy Rose Peter ${ }^{1 *}$, Jismon Sebastian ${ }^{2}$, Swapna S. Nair ${ }^{3}$ \\ ${ }^{1}$ M.Sc. Scholar, Department of Physics, Sacred Heart College, Thevara, India \\ ${ }^{2}$ M.Phil. Scholar, Department of Physics, Sacred Heart College, Thevara, India \\ ${ }^{3}$ Associate Professor, Department of Physics, Central University, Kasargod, India \\ *Corresponding author: emyredroses04@gmail.com
}

\begin{abstract}
Lead in our body is toxic and hazardous. Here leadfree Cobalt ferrite and Barium Titanate inks have been prepared and fabricated. The prepared inks remained stable without agglomeration or condensation during preservation. Cobalt Ferrite and Barium Titanate Nano inks have been characterized using X-ray diffraction method and UV Visible Spectroscopy. By the analysis of X-ray diffraction (XRD), the resultant inks were confirmed to be of pure Cobalt Ferrite and Barium Titanate powders with cubic structure and tetragonal structure respectively. Lattice parameters and grain size have been determined by X-ray diffraction method. UV Visible Spectroscopy analysis has been done to obtain the band gap energy of the prepared inks. The preparation and characterization of Cobalt Ferrite and Barium Titanate Nano inks are comprehensively demonstrated in this paper.
\end{abstract}

Keywords: Barium Titanate Nano ink, Cobalt Ferrite Nano ink, Inkjet printing.

\section{Introduction}

Usage of pigments containing lead in printing inks is widespread and is a common practice. The coloured pages of almost all newspapers and magazines contain high levels of lead. If we burn these papers containing lead, then this may result in air pollution and severe health problems like anaemia, weakness, kidney and brain damage in humans. Even small amounts of lead in the body are harmful. So there arises a need for having lead-free pigments in inks. Nanoparticles have more amplified properties than bulk solids. By making use of these properties in the ink industry we can make inks having more enhanced properties than that are available today. Such inks are called Nano inks. The size of the pigment used in making ink plays a major role. Their size can significantly affect the colour, strength and durability of the final product. Smaller the pigment size better will be the ink. Also, the particle size of the pigments must be small enough so that they do not block the nozzles of the printing mechanism. Decreasing the particle size will increase the stability of the ink. The colour of the ink depends on the pigment used and the particle size of the pigment governs the intensity of the ink colour. Thus we can see that Nano-sized particles can enhance the properties of the ink. This paper reports the preparation of Cobalt Ferrite and Barium Titanate Nano inks which are lead-free and cost-effective.

\section{Materials and Methods}

\section{A. Cobalt Ferrite}

Cobalt Ferrite is having inverse spinel structure. The magnetic properties such as saturation magnetization, remnant magnetization, coercivity and Curie temperature of Cobalt Ferrite at high temperatures are the same as at room temperature [1], [2].

\section{B. Barium Titanate}

This is an inorganic compound with the chemical formula $\mathrm{BaTiO}_{3} . \mathrm{BaTiO}_{3}$ is a ferroelectric ceramic material. They possess polarization in the absence of an electric field [2]. This is a white powder and transparent as larger crystals. It has $\mathrm{ABO} 3$ type perovskite structure. The cubic form of $\mathrm{BaTiO} 3$ is non-polar. By decreasing temperature below $120^{\circ} \mathrm{C}$, spontaneous polarization occurs in $\mathrm{BaTiO}_{3}$ and the crystal goes through a phase transition to the ferroelectric state. In this process, the cubic structure transforms to tetragonal. In the tetragonal form refractive index is independent of temperature. Barium Titanate is insoluble in water. Lead-free $\mathrm{BaTiO}_{3}$ remains attractive for environmental reasons [3]. Being a lead free ferroelectric ceramic, it is a good candidate for ink making.

\section{Synthesis of Cobalt Ferrite nanoparticles}

The sol of Cobalt Ferrite was prepared by a simple sol-gel method incorporating the nitrates of cobalt and iron (3+). Solgel is a chemical solution process used to make ceramic ink pigments and glass materials in the form of thin films or powders. A mixture of ethylene glycol and ethanol taken in the ratio 1:1 was used as the solvent and mixed with the nitrates of cobalt and iron (3+)[4]. The mixture was stirred until the gel was formed. The gel was dried in air at room temperature. The resulting solids were then ground and sieved to get fine powder which was annealed at $850^{\circ} \mathrm{c}$ for 3 hours. 


\section{IJRESM https://www.ijresm.com | ISSN (Online): 2581-5792 | RESAIM Publishing}

\section{Synthesis of Barium Titanate nanoparticles}

The sol-gel method is used for the preparation of Barium Titanate Nano powders. The reaction mixture solution was made of $6.80 \mathrm{ml}$ Butyl Titanate and $9.18 \mathrm{ml}$ of isopropanol after being stirred at room temperature for 0.5 hours. Then $3.43 \mathrm{ml}$ of glacial acetic acid was added to the mixed solution and stirred for 0.5 hours. The stability and viscosity of sol were optimized before making composite sol. The stabilized sol of Barium Titanate was heated at $60^{\circ} \mathrm{C}$ to get gel and then heat treatment is done at $200^{\circ} \mathrm{C}$ to burn out the organic solvent. This was then ground and annealed at $720^{\circ} \mathrm{c}$ to get fine Nano powders [5].

\section{E. Poly Vinyl Alcohol}

Poly Vinyl Alcohol (PVA) has high capability to bind pigments. PVA has a repeating vinyl alcohol unit with a molecular formula of [- $\mathrm{CH} 2 \mathrm{CHOH}-] \mathrm{n}$ [6]. It is a polymer which is soluble in water. Polymers are widely used in the present day inks to stabilize pigment dispersions. They attach onto the surface of the solid pigments by adsorption. Adsorbed layers of polymers tend to balance the attraction/repulsion forces among the pigment particles and prevent the flocculation and coagulation. Their film forming ability at a given temperature adds to the mechanical properties of the ink coating.

\section{F. Glycerol}

Glycerol also called Glycerine is a natural component of all animal and vegetable fats and oils. It can be synthesized from cane or corn syrup sugar or from substances such as Propylene [7], [8], [9]. Glycerol has three hydroxyl groups that are responsible for its solubility in water. Its chemical formula is $\mathrm{C}_{3} \mathrm{H}_{8} \mathrm{O}_{3}$.

\section{G. Preparation of Nano inks}

The Cobalt Ferrite Nano powder and Barium Titanate were taken in a separate mortar and pestle and drop by drop Poly Vinyl Alcohol and Glycerol were added simultaneously. They were mixed thoroughly until the ink consistency is obtained. Then ultra-sonication was done to dissolve all the particles completely into the solution. It was carried out for 5 to 15 minutes and then kept aside for few minutes. The prepared inks were characterized using X-ray diffraction and UV Visible absorption spectra. They were tried on black carbon paper, OHP paper and stamp paper to check whether they are suitable for printing purpose (Fig. 1).

\section{Results and Discussion}

\section{A. Structural and Optical characterization}

Fig. 2 shows X-ray diffractograms of Cobalt Ferrite and Barium Titanate Nano inks. In the case of Cobalt Ferrite, the prominent peaks correspond to miller indices (220), (311), (400), (422), (511), (440) are found at $2 \Theta$ values $30.208^{\circ}$, $35.542^{\circ}, 43.242^{\circ}, 53.980^{\circ}, 57.289^{\circ}$ and $62.644^{\circ}$ respectively
(Table 1). In the case of Barium Titanate, the prominent peaks correspond to miller indices (100), (101), (111), (200), (102), (112), (220), (212), (301), (311) and (222) are found at $2 \Theta$ values $22.37^{\circ}, 31.71^{\circ}, 38.95^{\circ}, 45.49^{\circ}, 51.172^{\circ}, 56.35^{\circ}, 65.953^{\circ}$, $70.611^{\circ}, 75.29^{\circ}, 79.7112^{\circ}$ and $83.58^{\circ}$ respectively (Table 2 ).

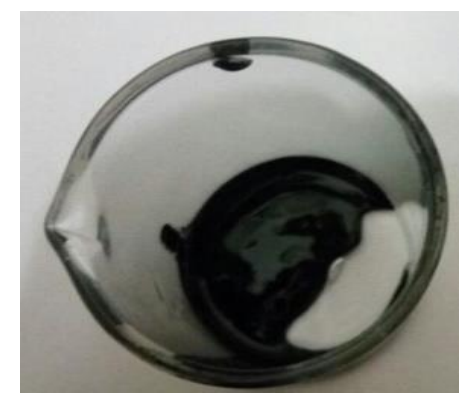

Prepared Cobalt ferrite Nano ink

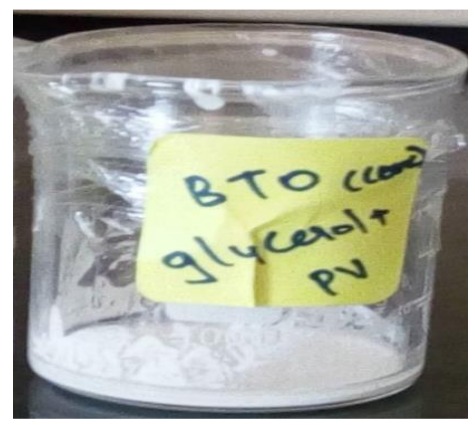

Prepared Barium Titanate Nano ink

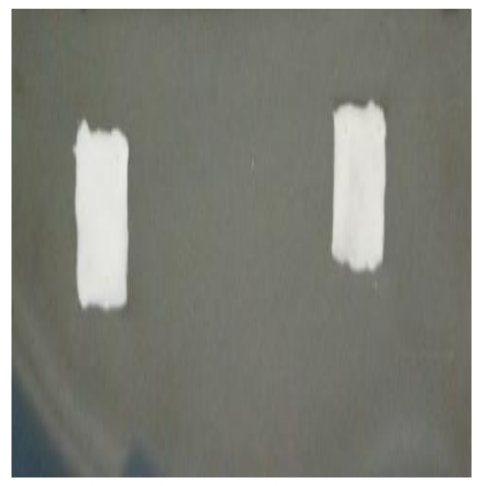

Barium Titanate on ink OHP sheet

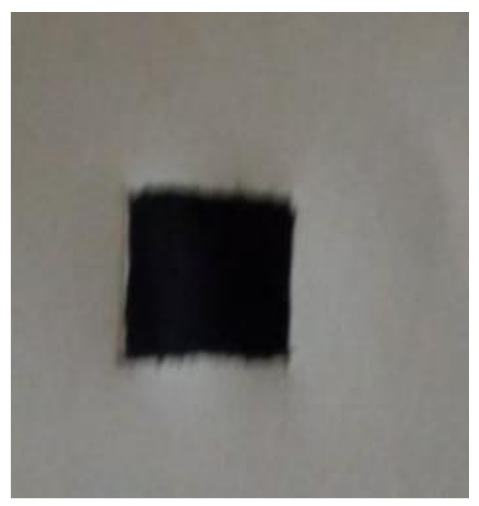

Cobalt Ferrite Nano on stamp paper 


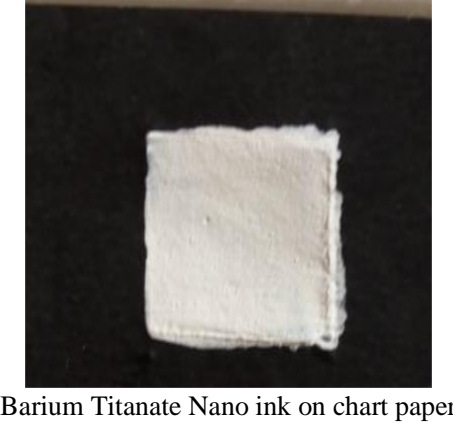

Fig. 1. Photographs of prepared inks tried on various surfaces

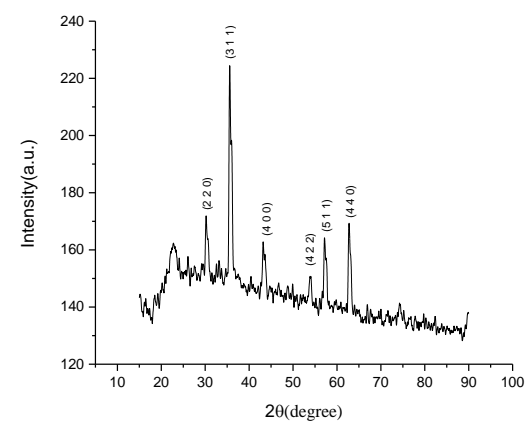

Fig. 2. XRD spectrum of Cobalt Ferrite Nano ink

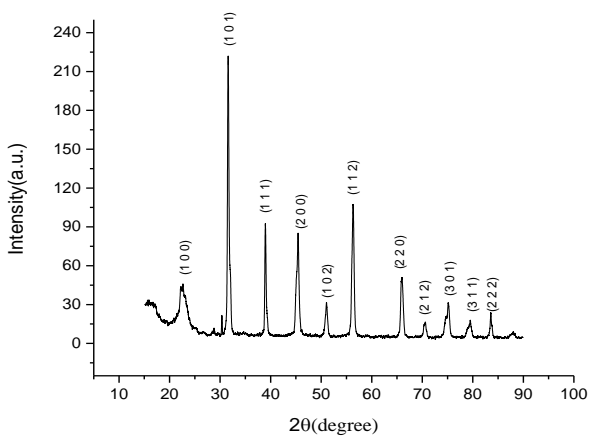

Fig. 3. XRD spectrum of Barium Titanate Nano ink

The grain size of Cobalt Ferrite and Barium Titanate Nano inks are determined by using Scherrer formula. Fig. 4 gives the full width half maxima (FWHM) of most intense peaks. The grain size of Cobalt Ferrite and Barium Titanate Nano inks are found to be $9.489 \mathrm{~nm}$ and $21.577 \mathrm{~nm}$ respectively.

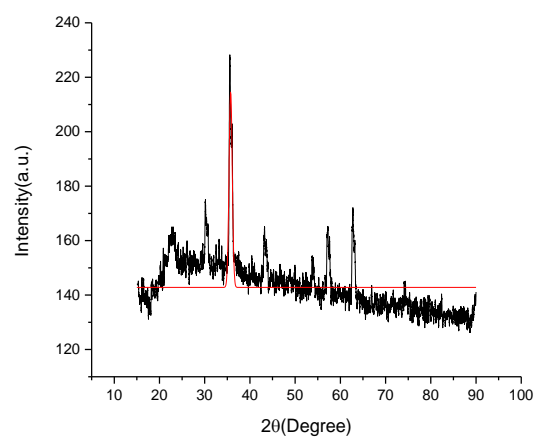

Fig. 4. FWHM of Cobalt Ferrite Nano ink

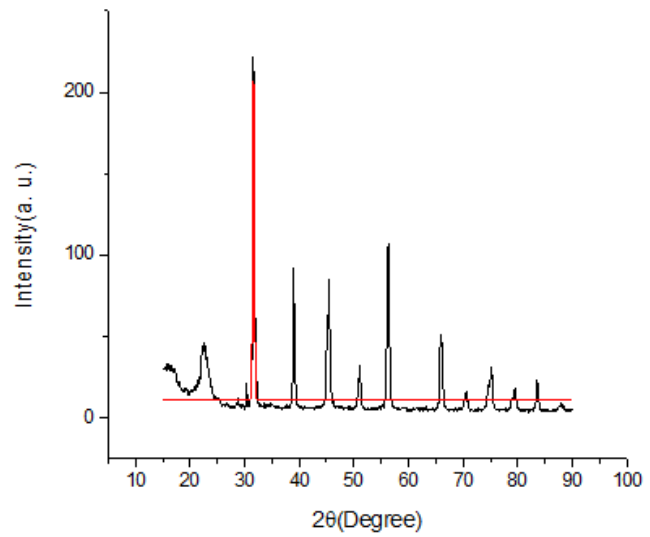

Fig. 5. FWHM of Barium Titanate Nano ink

Table 1

XRD data of Cobalt Ferrite Nano ink

\begin{tabular}{|l|l|l|l|}
\hline $2 \Theta($ degree $)$ & $\Theta($ degree $)$ & $\mathrm{d}$ & Miller indices \\
\hline 30.208 & 15.104 & 2.9562 & $(220)$ \\
35.542 & 17.771 & 2.5238 & $(311)$ \\
43.292 & 21.646 & 2.08826 & $(400)$ \\
53.980 & 26.99 & 1.6731 & $(422)$ \\
57.289 & 28.6445 & 1.60688 & $(511)$ \\
62.644 & 31.322 & 1.48178 & $(440)$ \\
\hline
\end{tabular}

For cubic structure the spacing between the lattice planes and lattice constants are related by the formula [9],

$$
1 / d^{2}=\left(h^{2}+k^{2}+l^{2}\right) / a^{2}
$$

where $\mathrm{d}$ is the interplanar spacing, a is the lattice constant, $\mathrm{h}$, $\mathrm{k}$ and $\mathrm{l}$ are the miller indices of the plane.

The obtained lattice parameters are: $a=b=c=8.3529 \mathrm{~A}^{\mathrm{O}}$

Table 2

XRD data of Barium Titanate Nano ink

\begin{tabular}{|l|l|l|l|}
\hline $2 \Theta($ in degree) & $\Theta$ (in degree) & $\mathrm{d}$ & Miller indices \\
\hline 22.37 & 11.185 & 3.971 & $(100)$ \\
31.71 & 15.855 & 2.8195 & $(101)$ \\
38.95 & 19.475 & 2.31046 & $(111)$ \\
45.49 & 22.723 & 1.99417 & $(200)$ \\
51.172 & 25.586 & 1.7837 & $(102)$ \\
56.35 & 28.175 & 1.63142 & $(112)$ \\
65.953 & 32.976 & 1.4152 & $(220)$ \\
70.611 & 35.3059 & 1.3328 & $(212)$ \\
75.29 & 37.645 & 1.2612 & $(301)$ \\
79.7112 & 39.8556 & 1.2019 & $(311)$ \\
83.58 & 41.79 & 1.1559 & $(222)$ \\
\hline
\end{tabular}

For tetragonal structure the spacing between the lattice planes and lattice constants are related by the formula [9],

$$
1 / d^{2}=h^{2} / a^{2}+k^{2} / b^{2}+l^{2} / c^{2}
$$

The obtained lattice parameters are:

$$
\begin{aligned}
& \mathrm{a}=\mathrm{b}=3.971 \mathrm{~A}^{\mathrm{o}} \\
& \mathrm{c}=4.00396 \mathrm{~A}^{\mathrm{o}}
\end{aligned}
$$




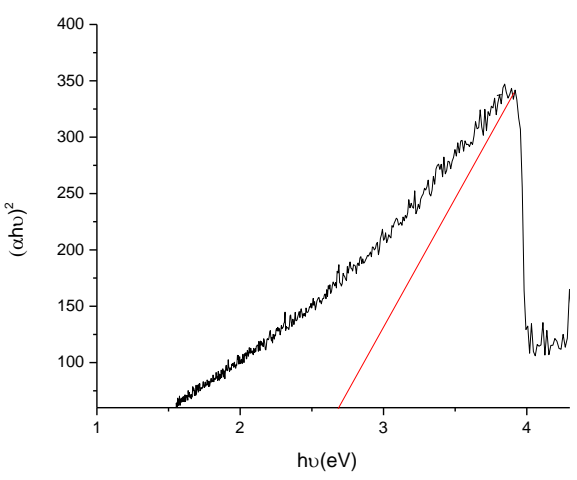

Fig. 6. Tauc plot of Cobalt Ferrite Nano ink

Fig. 6, show the tauc plot obtained from UV Visible spectrum analysis from which the band gap of both the inks has been calculated.

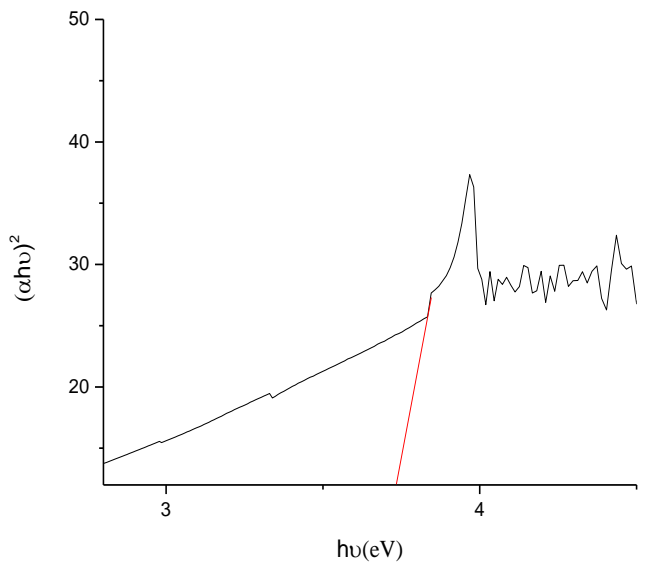

Fig. 7. Tauc plot of Barium Titanate nano ink
Band gap energy of Cobalt Ferrite Nano ink was found to be $2.68856 \mathrm{eV}$ and band gap energy of Barium Titanate Nano ink was found to be $3.7357 \mathrm{eV}$.

\section{Conclusion}

In the present study Cobalt Ferrite and Barium Titanate Nano inks were prepared. These inks were characterized by X-Ray Diffraction method and UV Visible Spectroscopy. The structure, lattice parameters and grain size of both the inks were analyzed using X-Ray Diffraction and band gap energy using UV Visible Spectroscopy. The structure of the Cobalt Ferrite Nano ink is cubical and the grain size is $9.489 \mathrm{~nm}$. The structure of Barium Titanate Nano ink is tetragonal and the grain size is $21.577 \mathrm{~nm}$. The bandgap energy of Cobalt ferrite Nano ink is $2.6885 \mathrm{eV}$ and that of Barium Titanate Nano ink is $3.7357 \mathrm{eV}$. Initial step of preparation of cost effective lead free Nano ink was successful and was able to fabricate.

\section{References}

[1] https://www.slideshare.net/icaer2013/291-sarita

[2] https://www.adichemistry.com/inorganic/cochem/spinels/spinelstructures.html

[3] https://www.britannica.com/science/Ethyleneglycol

[4] I. Kanno, T. Ichida, K. Adachi, H. Kotera, K. Shibata, T. Mishimka, "Power generation performance of lead-free $(\mathrm{K}, \mathrm{Na}) \mathrm{Nbo}_{3}$ piezoelectric thin-film energy harvestors. Sensors and Actuators" Physica A:, vol. 179, pp.132-136, 2012

[5] X. C. Li, Q. Yang, "Research on Preparation of Barium Titanate Nano Powder by Sol-Gel", Materials Science Forum, Vol. 809-810, pp. 136139, 2015.

[6] https://www.britannica.com/science/polyvinyl-alcohol

[7] K. Reeta, "Glycerol - Definition of glycerol in English by Oxford Dictionaries". Oxford Dictionaries - English. Christoph, Ralf; Schmidt, Bernd; Steinberner, Udo; Dilla, Wolfgang; (2006).

[8] "Glycerol". Ullmann's Encyclopedia of Industrial Chemistry. Ullmann's Encyclopedia of Industrial Chemistry.

[9] H. Hirschmann, "The Nature of Substrate Asymmetry in Stereoselective Reactions", Journal of Biological Chemistry, vol. 235, pp. 2762-2767, 1960. 